\title{
SOME INEQUALITIES FOR THE GENERALIZED SINE AND THE GENERALIZED HYPERBOLIC SINE
}

\author{
Li YIN AND LI-GUO HUANG
}

Abstract. In this paper, the authors establish some new inequalities for the generalized sine and the generalized hyperbolic sine.

Mathematics subject classification (2010): 26A51, 26D05, 33B10.

Keywords and phrases: Generalized sine, generalized hyperbolic sine, inverse function, inequalities.

\section{REFERENCES}

[1] G. D. Anderson, M. K. Vamanamurthy and M. Vuorinen, Topics in special functions, Papers on Analysis: A volume dedicated to Olli Martio on the occasion of his 60th birthday, ed. by J. Heinonen, T. Kilpeläinen, and P. Koskela, Report 83, Univ. Jyväskylä (2001), 5-26, available online at http://arxiv.org/abs/0712.3856.

[2] G. D. Anderson, M. K. Vamanamurthy and M. Vuorinen, Special functions of quasiconformal theory, Expo. Math., 7 (1989), 97-136.

[3] G. D. Anderson, M. Vuorinen AND X.-H. Zhang, Topics in special functions III, available online at http://arxiv.org/abs/1209.1696.

[4] B. A. Bhayo And M. Vuorinen, Power mean inequality of generalized trigonometric functions, available online at http://arxiv.org/abs/1209.0873.

[5] B. A. BHAYO AND M. VuORINEN, On generalized trigonometric functions with two parameters, J. Approx. Theory 164 (2012), no. 10, 1415-1426, available online at http://arxiv.org/abs/ 1112.0483.

[6] B. A. Bhayo And M. Vuorinen, Inequalities for eigenfunctions of the p-Laplacian, available online at http://arxiv.org/abs/1101.3911.

[7] W.-D. JIANG, M.-K. WANG, Y.-M. ChU, Y.-P. JIANG, AND F. QI, Convexity of the generalized sine function and the generalized hyperbolic sine function, J. Approx. Theory, 174 (2013), 1-9, available online at http://arxiv.org/abs/1301.3264.

[8] R. KlÉn, M. VuORINEN AND X.-H. ZHANG, Inequalities for the generalized trigonometric and hyperbolic functions, J. Math. Anal. Appl., 409 (2014), no. 1, 521-529, available online at http://arxiv.org/abs/1210.6749.

[9] P. Lindqvist, Some remarkable sine and cosine functions, Ricerche di Matematica Vol. XLIV (1995), 269-290.

[10] D. S. Mitrinović, Analytic inequalities, Springer Verlag, New York, 1970.

[11] J. SÁNDOR, On certain inequalities for hyperbolic and trigonometric functions, J. Math. Inequal. 7 (2013), no. 3, 241-245. 